\title{
Integrated vs. sequential reaction and separation: contributions for a global analysis
}

\author{
Ana L. Paiva, Diman van Rossum, F. Xavier Malcata* \\ Escola Superior de Biotecnologia, Universidade Catolica Portuguesa, Rua Dr. António Bernardino de Almeida, P-4200 Porto, Portugal
}

\begin{abstract}
Integration of reaction and separation in one single step has often been claimed to provide enhanced processing and economic results when compared with the traditional configuration where a reaction unit is followed by a downstream separation unit, due to alleviation of kinetic and thermodynamic constraints. This paper quantitatively addresses the kinetic and thermodynamic improvements which can be brought about by performing reaction and separation simultaneously instead of sequentially, in the case of a unisubstrate/uniproduct reversible reaction following first-order kinetics and that takes place in a system behaving as an ideal solution. Kinetic enhancement was ascertained via theoretical evolution of the molar fraction of product in both streams coming from either the separator (in series with the reactor) or from the integrated unit, whereas thermodynamic enhancement was ascertained via theoretical evolution of the overall Gibbs' free energy in either configuration. The time required to achieve a predefined degree of conversion and separation is always lower for simultaneous than for sequential reaction and separation. The molar fraction of product in the product-rich stream is always higher for the integrated unit except for high values of parameter $\phi$ (defined as the ratio of the time scale associated with chemical reaction to the time scale associated with mass transfer of reactant) and of the chemical equilibrium constant. Comparison of the thermodynamic behaviour of both systems also leads to the conclusion that high values of $\phi$ yield worse results when the integrated unit is used instead of the sequential reactor/separator system because reactant is removed from the reacting system at a rate that is higher than the reaction rate itself.
\end{abstract}

Keywords: Chemical reaction; Physical separation; Unit operations; Thermodynamics; Kinetics

\section{Introduction}

Chemical processes have traditionally been carried out in reaction units followed by separation units; the latter are required because kinetic and/or thermodynamic constraints do not allow the reaction approach completion within a given time frame, with concomitant contamination of products with unreacted reactants. However, since the phenomena of mixing, chemical transformation and transport occur on the molecular scale, the issue of integration of reaction and separation (i.e. performing reaction and separation simultaneously in one single step) has for some time been under the scrutiny of several researchers (e.g. Paiva and Malcata, 1994, 1996, 1997a-c, 1999; Paiva et al., 1998). Integration apparently alleviates a series of limiting factors that are quite common in biochemical reactions, e.g. high product inhibi-

\footnotetext{
*Corresponding author. Tel.: 3512 5580004; fax: 35125090351.
}

tion and low volumetric productivity. Furthermore, the possibility of continuously and immediately removing the product(s) formed by reaction improves effectiveness of separation because bulk high concentrations are not allowed to build up, thus reducing the costs usually associated with post-reaction separation.

Kinetics and thermodynamics play important roles in both the reaction and separation steps. Laane et al. (1987) and Tramper et al. (1992) have claimed that reaction coupled with in situ separation brings about kinetic enhancements, especially in the case of biochemical processes. However, such claim tends only to consider the point of view of the reaction rather than the point of view of the overall process, constituted by reaction and separation. On the other hand, Paiva and Malcata (1997b) have demonstrated that integration of reaction and separation does not actually affect the overall thermodynamics of the system if the Gibbs' free energy is used as a quantitative measure because it is a state function (and, thus, independent of path). 
This paper attempts to quantitatively address the issue of kinetic and thermodynamic enhancements which are tentatively brought about by performing reaction and separation in a simultaneous rather than in a sequential fashion. The model system selected consists in a reaction that follows a 1:1 stoichiometry and evolves from pure reactant $(\mathrm{R})$ to a thermodynamically ideal reactant/product homogeneous mixture $(\mathrm{R}+\mathrm{P})$, according to the following mechanism that encompasses only elementary steps:

$\left[\mathbf{R} \underset{k_{-1}}{\stackrel{k_{1}}{\rightleftharpoons}} \mathbf{P}\right]$

where $k_{1}$ and $k_{-1}$ are the first-order kinetic constants describing the rate of reaction in the forward and reverse direction, respectively, and where, for the sake of simplicity, only first-order kinetics will be considered. Selection of a simple binary system agrees with the current trend towards substitution of dilute solutions by solvent-free mixtures, thus increasing concentration of reactant to the highest possible level, and consequently obtaining a higher degree of conversion and avoiding higher downstream separation problems. The alternative configurations of independent reactor and separator (see Fig. 1) and integrated reactor and separator (see Fig. 2) will be the focus of our attention for such general reversible reaction in terms of both product molar fraction and Gibbs' free energy profiles.

\section{Kinetic evolution}

\subsection{Sequential reaction and separation}

\subsubsection{Reaction step}

Considering the model reaction described above, the mass balances to reactant $\mathrm{R}$ and product $\mathrm{P}$ in a reactor in the absence of separation read

$\frac{\mathrm{d} n_{R}}{\mathrm{~d} t}=-k_{1} C_{R}+k_{-1} C_{P} \quad$ at $t=0, \quad n_{R, 0}=n_{0}$,

$\frac{\mathrm{d} n_{P}}{\mathrm{~d} t}=k_{1} C_{R}-k_{-1} C_{P} \quad$ at $t=0, \quad n_{P, 0}=0$,

where $n_{R}$ and $n_{P}$ denote the number of moles of $\mathrm{R}$ and $\mathrm{P}$, respectively, $n_{R, 0}$ and $n_{P, 0}$ the initial number of moles of $\mathrm{R}$ and $\mathrm{P}$, respectively, $t$ the time elapsed after startup in the case of a batch reactor, or the space time in the case of a plug-flow reactor, $C_{R}$ and $C_{P}$ the molar concentrations of $\mathrm{R}$ and $\mathrm{P}$, respectively, and $n_{0}$ the initial number of moles of reactant. Recalling that $V=v\left(n_{R}+n_{P}\right)$, where $V$ is the total volume and $v$ is the molar volume of either $\mathrm{R}$ or $\mathrm{P}$ (which, for the sake of simplicity but without loss of generality, will be considered to be equal to one another), coupled with $C_{R}=n_{R} / V$ and $C_{P}=n_{P} / V$, Eq. (1a)

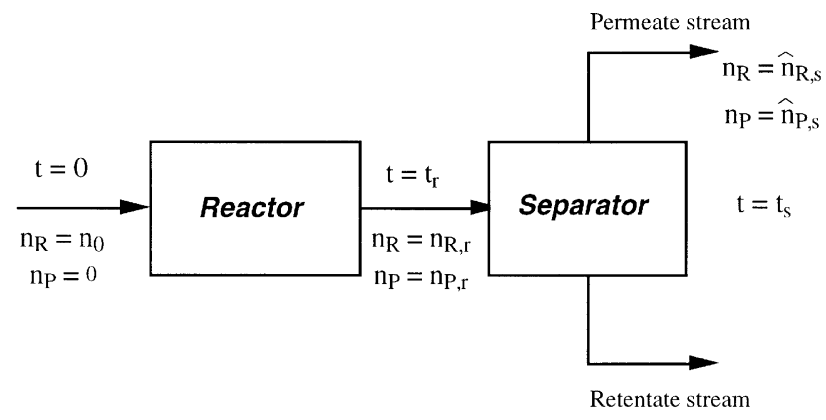

$\mathrm{n}_{\mathrm{R}}=\mathrm{n}_{\mathrm{R}, \mathrm{s}}$ $n_{P}=n_{P, s}$

Fig. 1. Schematic representation of the classical configuration consisting of a reaction unit in series with a separation unit.

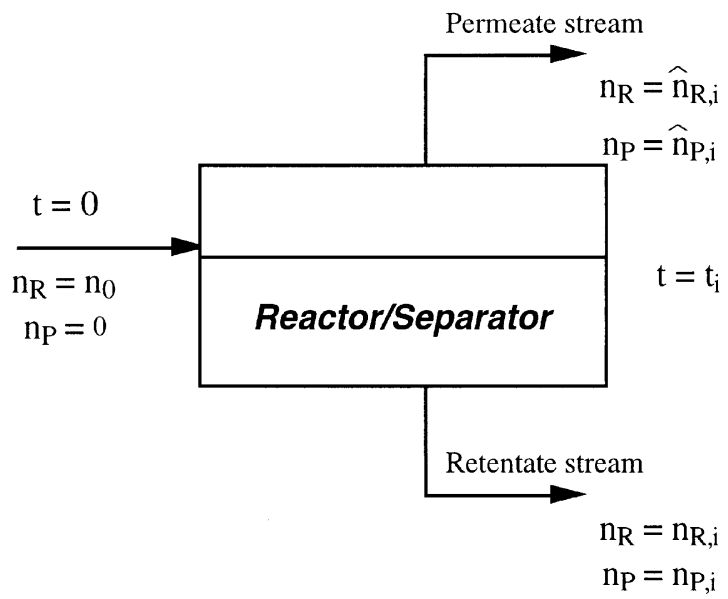

Fig. 2. Schematic representation of the modern configuration consisting of an integrated reaction/separation unit.

becomes

$$
\begin{aligned}
\frac{\mathrm{d} n_{R}}{\mathrm{~d} t} & =-\frac{1}{v n_{0}}\left(k_{1} n_{R}-k_{-1} n_{P}\right) \\
\text { at } t & =0, \quad n_{R, 0}=n_{0}, \quad n_{P, 0}=0,
\end{aligned}
$$

where advantage was taken from the $1: 1$ stoichiometry of the chemical reaction to replace $\left(n_{R}+n_{P}\right)$ by $n_{0}$. Algebraic combination of Eqs. (1a) and (1b) yields

$$
\begin{aligned}
& \frac{\mathrm{d} n_{R}}{\mathrm{~d} t}+\frac{\mathrm{d} n_{P}}{\mathrm{~d} t}=0 \\
& \text { at } t=0, \quad n_{R, 0}=n_{0}, \quad n_{P, 0}=0 .
\end{aligned}
$$

Taking advantage of the fact that in the reactor, at any moment,

$n_{P}=n_{0}-n_{R}$,

and recalling that $K_{\text {eq }}=k_{1} / k_{-1}=C_{P, \text { eq }} / C_{R, \text { eq }}=$ $n_{P, \text { eq }} / n_{R \text {, eq }}$ (where $K_{\text {eq }}$ denotes the equilibrium constant and subscript eq denotes equilibrium conditions), 
integration of Eq. (2b) coupled with Eq. (2a) finally gives

$\frac{\mathrm{d} n_{R}}{\mathrm{~d} t}=\frac{k_{1}}{v n_{0}}\left(\frac{n_{0}}{K_{\mathrm{eq}}}-\left(1+\frac{1}{K_{\mathrm{eq}}}\right) n_{R}\right)$

at $t=0, \quad n_{R, 0}=n_{0}$.

Eqs. (3a) and (3b) can be arranged to read

$\frac{\mathrm{d} n_{R}^{*}}{\mathrm{~d} t^{*}}=\frac{1}{K_{\mathrm{eq}}}-\left(1+\frac{1}{K_{\mathrm{eq}}}\right) n_{R}^{*}$

at $t^{*}=0, \quad n_{R, 0}^{*}=1$,

$n_{P}^{*}=1-n_{R}^{*}$,

where the dimensionless variables are defined as

$t^{*}=\frac{k_{1} t}{v n_{0}}$,

$n_{R}^{*}=\frac{n_{R}}{n_{0}}$

$n_{P}^{*}=\frac{n_{P}}{n_{0}}$.

Integration of Eq. (4a) finally gives

$n_{R}^{*}=\frac{1+K_{\mathrm{eq}} \exp \left\{-\left[\left(1+K_{\mathrm{eq}}\right) / K_{\mathrm{eq}}\right] t^{*}\right\}}{1+K_{\mathrm{eq}}}$,

whereas combination of Eq. (4b) with Eq. (6) gives

$n_{P}^{*}=\frac{K_{\mathrm{eq}}\left(1-\exp \left\{-\left[\left(1+K_{\mathrm{eq}}\right) / K_{\mathrm{eq}}\right] t^{*}\right\}\right)}{1+K_{\mathrm{eq}}}$.

If one assumes that the reaction is allowed to proceed until chemical equilibrium is reached, then the composition at equilibrium will be obtained from Eqs. (6) and (7) as the limit when $t^{*}$ tends to infinity, viz.

$n_{R, \mathrm{eq}}^{*}=\frac{1}{1+K_{\mathrm{eq}}}, \quad n_{P, \mathrm{eq}}^{*}=\frac{K_{\mathrm{eq}}}{1+K_{\mathrm{eq}}}$.

Since achievement of equilibrium would require an infinite time, then one may assume that reaction will be allowed to proceed only until a predefined neighbourhood of equilibrium is reached or, equivalently, until $n_{P, r}^{*}=\chi n_{P, \text { eq }}^{*}$, where subscript $r$ denotes the end of the reaction step, and where parameter $\chi$, which describes the fraction of the overall number of moles of product $\mathrm{P}$ if chemical equilibrium were attained, accordingly takes values (slightly) below unity. The time it would take to achieve such extent of reaction will be obtained from combination of Eqs. (6) and (8) as

$$
\begin{aligned}
& n_{R}^{*}=n_{R, r}^{*}=\frac{1+(1-\chi) K_{\mathrm{eq}},}{1+K_{\mathrm{eq}}}, \\
& n_{P}^{*}=n_{P, r}^{*}=\frac{\chi K_{\mathrm{eq}}}{1+K_{\mathrm{eq}}}, \\
& \quad \text { at } t^{*}=t_{r}^{*}=-\frac{K_{\mathrm{eq}}}{1+K_{\mathrm{eq}}} \ln (1-\chi) .
\end{aligned}
$$

\subsubsection{Separation step}

Consider now the separation step which occurs when the outlet stream from the reactor becomes the inlet stream to the separator (see Fig. 1). Two outlet streams will normally form in said separating unit: a retentate stream (i.e. the R-rich, or lower outlet stream), and a permeate stream (i.e. the P-rich, or upper outlet stream). If a sweep stream, available at a very high volumetric flow rate, carries away the compounds that have just left the retentate stream (as would happen during evaporation using a sweep gas in the vapour phase), or alternatively, if the permeate stream drips out from the separator (as would happen during percolation through a molecular sieve), then the concentration of both components in the permeate can, at all times, be considered to be virtually nil; therefore, the mass balances to $\mathrm{R}$ and $\mathrm{P}$ in the retentate stream of the separator in the absence of reaction will simply read

$$
\begin{aligned}
& \frac{\mathrm{d} n_{R}}{\mathrm{~d} t}=-k_{R} C_{R} \\
& \quad \text { at } t=t_{r}, \quad n_{R}=\left(1+(1-\chi) K_{\mathrm{eq}}\right) n_{R, \mathrm{eq}},
\end{aligned}
$$

$\frac{\mathrm{d} n_{P}}{\mathrm{~d} t}=-k_{P} C_{P}$

$$
\text { at } t=t_{r}, \quad n_{P}=\chi n_{P, \text { eq }},
$$

where $t$ is the time elapsed after startup of the reactor in the case of a batch separator, or the space time in the case of a plug flow separator, and $k_{R}$ and $k_{P}$ the first-order kinetic constants describing the rate of mass transfer of $\mathrm{R}$ and $\mathrm{P}$, respectively. Recalling again that $V=v\left(n_{R}+n_{P}\right)$ coupled with $C_{R}=n_{R} / V$ and $C_{P}=n_{P} / V$, Eqs. (10a) and (10b) become

$$
\begin{aligned}
\frac{\mathrm{d} n_{R}}{\mathrm{~d} t} & =-\frac{k_{R}}{v} \frac{n_{R}}{n_{R}+n_{P}} \\
\text { at } t & =t_{r}, \\
n_{R} & =n_{R, r}=\left(1+(1-\chi) K_{\mathrm{eq}}\right) n_{R, \text { eq }}, \\
\frac{\mathrm{d} n_{P}}{\mathrm{~d} t} & =-\frac{k_{P}}{v} \frac{n_{P}}{n_{R}+n_{P}} \\
\text { at } t & =t_{r}, \quad n_{P}=n_{P, r}=\chi n_{P, \text { eq }} .
\end{aligned}
$$

Algebraic combination of Eqs. (11a) and (11b) allows Eq. (11b) to be replaced by

$$
\begin{aligned}
& \frac{\mathrm{d} n_{P}}{\mathrm{~d} n_{R}}=\frac{k_{P}}{k_{R}} \frac{n_{P}}{n_{R}} \\
& \text { at } n_{R, r}=\left(1+(1-\chi) K_{\text {eq }}\right) n_{R, \text { eq }}, \\
& n_{P, r}=\chi n_{P, \text { eq }}
\end{aligned}
$$


which, upon integration, is equivalent to

$n_{P}=\frac{\chi n_{P, \mathrm{eq}}}{\left(\left(1+(1-\chi) K_{\mathrm{eq}}\right) n_{R, \mathrm{eq}}\right)^{\alpha}} n_{R}^{\alpha}$.

Here $\alpha$ is a dimensionless parameter defined by

$\alpha=\frac{k_{P}}{k_{R}}$

and, based on the assumption that $\mathrm{P}$ is preferentially removed when compared with $\mathrm{R}, \alpha$ should take values above unity. Combination of Eq. (11a) with Eq. (13) gives

$\frac{\mathrm{d} n_{R}}{\mathrm{~d} t}=-\frac{k_{R}}{v} \frac{n_{R}}{n_{R}+\chi n_{P, \mathrm{eq}} /\left[\left(\left(1+(1-\chi) K_{\mathrm{eq}}\right) n_{R, \mathrm{eq}}\right)^{\alpha}\right] n_{R}^{\alpha}}$

at $t=t_{r}, \quad n_{R}=n_{R, r}=\left(1+(1-\chi) K_{\mathrm{eq}}\right) n_{R, \mathrm{eq}}$

or, upon combination with Eqs. (5), (8) and (9),

$\frac{\mathrm{d} n_{R}^{*}}{\mathrm{~d} t^{*}}=$

$$
\begin{gathered}
-\frac{\phi}{1+\left\{\left[\chi K_{\mathrm{eq}}\left(1+K_{\mathrm{eq}}\right)^{\alpha-1}\right] /\left[\left(1+(1-\chi) K_{\mathrm{eq}}\right)^{\alpha}\right]\right\} n_{R}^{* \alpha-1}} \\
\text { at } t^{*}=-\frac{K_{\mathrm{eq}}}{1+K_{\mathrm{eq}}} \ln \{1-\chi\}, \quad n_{R}^{*}=\frac{1+(1-\chi) K_{\mathrm{eq}}}{1+K_{\mathrm{eq}}},
\end{gathered}
$$

$$
n_{P}^{*}=\frac{\chi K_{\mathrm{eq}}\left(1+K_{\mathrm{eq}}\right)^{\alpha-1}}{\left(1+(1-\chi) K_{\mathrm{eq}}\right)^{\alpha}} n_{R}^{* \alpha},
$$

where the dimensionless parameter $\phi$ is defined as

$\phi=\frac{k_{R}}{k_{1}}$. calculated through

$\hat{n}_{R}^{*}=\frac{1+(1-\chi) K_{\mathrm{eq}}}{1+K_{\mathrm{eq}}}-n_{R}^{*}$,

$\hat{n}_{P}^{*}=\frac{\chi K_{\mathrm{eq}}}{1+K_{\mathrm{eq}}}-n_{P}^{*}$,

where $\hat{n}_{R}^{*}$ and $\hat{n}_{P}^{*}$ are the normalized numbers of moles of $\mathrm{R}$ and $\mathrm{P}$, respectively.

Since complete mass transfer between the retentate and permeate streams would not lead to any separation at all (except in the case of $\alpha=\infty$ ), then one may assume that separation will be allowed to proceed only until a predefined neighbourhood of total recovery of $\mathrm{P}$ is reached, or, equivalently, until $\hat{n}_{P, s}^{*}=\xi \hat{n}_{P, r}^{*}$, where subscript $s$ denotes the end of the separation step, and where parameter $\xi$, which describes the fraction of the overall number of moles of product $\mathrm{P}$ if full recovery were attained, accordingly takes values (slightly) below unity. Since, as demonstrated by Paiva et al. (1998), the most interesting and informational results when comparing integration processes with sequential reaction and separation processes are obtained when the time scales and the extents of reaction and separation are of the same order of magnitude (i.e. the process is neither reaction- nor separation-controlled), it will hereafter be considered that $\xi=\chi$ (which will dramatically reduce the number of possible situations by eliminating one degree of freedom). Under this assumption, the time it will take to achieve such extent of separation can then be obtained from Eqs. (16b) and (18) as

$$
t_{s}^{*}=-\frac{K_{\mathrm{eq}}}{1+K_{\mathrm{eq}}} \ln \{1-\chi\}+\frac{K_{\mathrm{eq}} \chi^{2}+\alpha\left[1+(1-\chi) K_{\mathrm{eq}}(1-\sqrt[\alpha]{1-\chi})-\sqrt[\alpha]{1-\chi}\right]}{\alpha \phi\left(1+K_{\mathrm{eq}}\right)} .
$$

Integration of Eq. (16a) finally gives the total time elapsed after reaction followed by separation (where $n_{r}^{*}$ denotes the dimensionless number of moles of $\mathrm{R}$ in the retentate stream), viz.

$$
\begin{aligned}
t^{*}= & -\frac{K_{\mathrm{eq}}}{1+K_{\mathrm{eq}}} \ln \{1-\chi\} \\
& +\frac{1}{\phi}\left(\frac{\chi K_{\mathrm{eq}}}{\alpha\left(1+K_{\mathrm{eq}}\right)}+\frac{1+(1-\chi) K_{\mathrm{eq}}}{1+K_{\mathrm{eq}}}-n_{R}^{*}\right. \\
& \left.-\frac{\chi K_{\mathrm{eq}}\left(1+K_{\mathrm{eq}}\right)^{\alpha-1}}{\alpha\left(1+(1-\chi) K_{\mathrm{eq}}\right)^{\alpha}} n_{R}^{* \alpha}\right) .
\end{aligned}
$$

The composition of the permeate (i.e. the P-rich, or the upper outlet) stream in the separator (see Fig. 1) may be
For the reactor/separator sequential configuration, the variation of the molar fraction of $\mathrm{P}, x_{P}^{*}$, given by $n_{P}^{*} /\left(n_{R}^{*}+n_{P}^{*}\right)$, is represented in Figs. $3 \mathrm{a}-\mathrm{c}$ as a function of the dimensionless time, $t^{*}$, until $t_{r}^{*}$ is reached, under the assumptions that $K_{\text {eq }}=1$ and that reaction proceeds until $95 \%$ of the equilibrium conversion is reached, i.e. $\chi=0.95$. For values of $t^{*}$ above $t_{r}^{*}$, the variation of $x_{P}^{*}$ in the retentate stream, given by $n_{P}^{*} /\left(n_{R}^{*}+n_{P}^{*}\right)$, and of $\hat{x}_{P}^{*}$ in the permeate stream, given by $\hat{n}_{P}^{*} /\left(\hat{n}_{R}^{*}+\hat{n}_{P}^{*}\right)$, are also represented in the same graph as two different lines as a function of $t^{*}$, again under the assumptions that $K_{\mathrm{eq}}=1$ and $\xi=\chi=0.95$, for different combinations of parameters $\phi$ and $\alpha$.

\subsection{Simultaneous reaction and separation}

In the case of the integrated unit, where reaction and separation occur simultaneously, the mass balances to $\mathrm{R}$ and $\mathrm{P}$ in the retentate stream of an integrated 

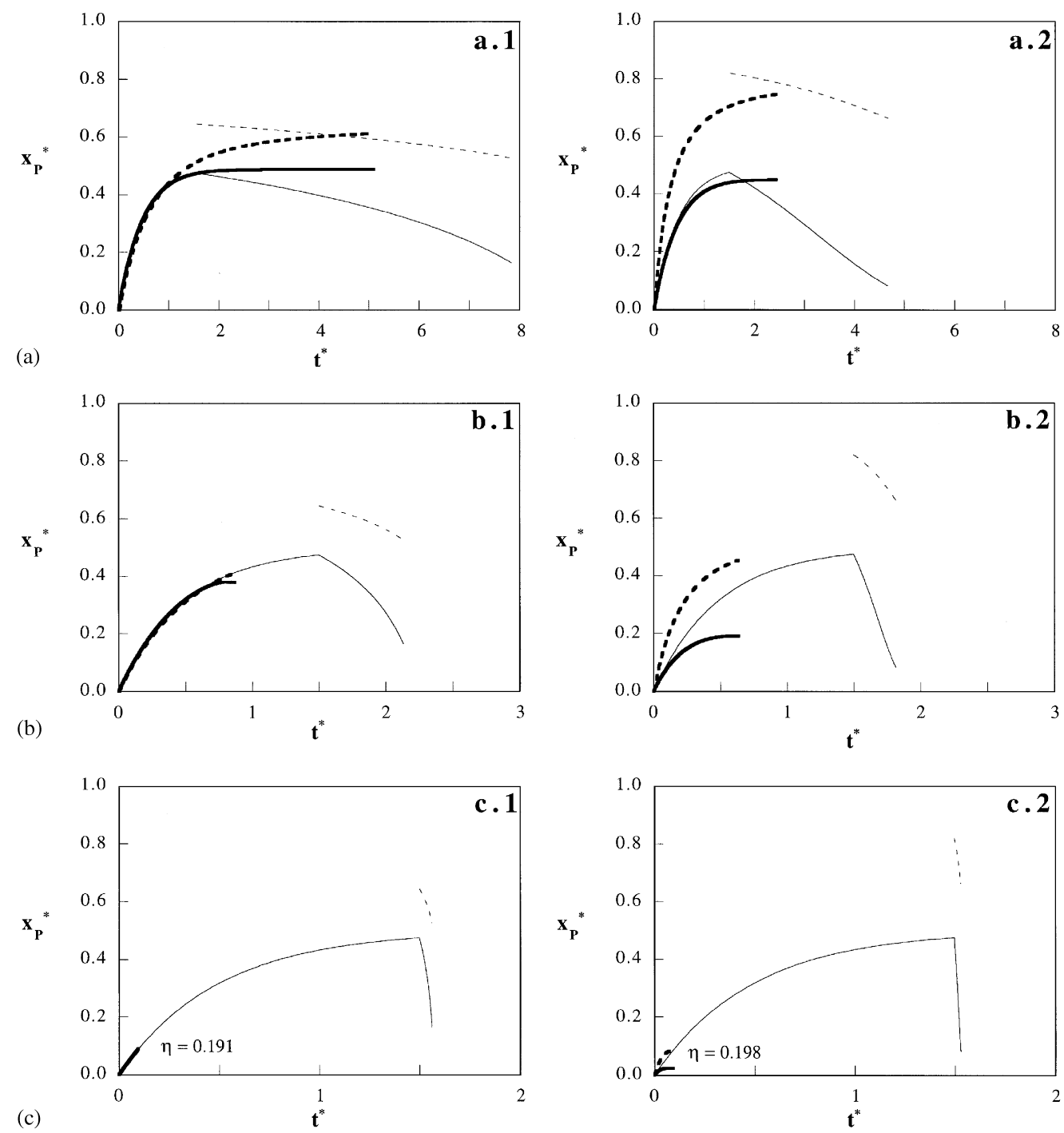

Fig. 3. Composition profiles of retentate $(-)$ and permeate streams $(---)$ at the outlet of the separator in the case of sequential reaction and separation (thin lines), or at the outlet of the integrated unit in the case of simultaneous reaction and separation (thick lines), expressed as the molar fractional amount of $\mathrm{P}, x_{P}^{*}$, vs. the dimensionless time, $t^{*}$, for $K_{\mathrm{eq}}=1$ and (a) $\phi=0.1$, (b) $\phi=1$ and (c) $\phi=10$, and for (1) $\alpha=2$ and (2) $\alpha=5$.

reactor/separator system read

$$
\frac{\mathrm{d} n_{R}}{\mathrm{~d} t}=-k_{1} C_{R}+k_{-1} C_{P}-k_{R} C_{R}
$$$$
\text { at } t=0, \quad n_{R}=n_{0},
$$

$$
\frac{\mathrm{d} n_{P}}{\mathrm{~d} t}=k_{1} C_{R}-k_{-1} C_{P}-k_{P} C_{P}
$$

$$
\text { at } t=0, \quad n_{P}=0,
$$

where $t$ is the time elapsed after startup in the case of a batch reactor/separator, or the space time in the case of a plug flow reactor/separator. Recalling again that $V=v\left(n_{R}+n_{P}\right), C_{R}=n_{R} / V, C_{P}=n_{P} / V$ and $K_{\text {eq }}=k_{1} /$ $k_{-1}=C_{P, \text { eq }} / C_{R, \text { eq }}=n_{P, \text { eq }} / n_{R \text {, eq }}$, coupled with Eqs. (5), (14) and (17), Eq. (21) becomes

$$
\begin{aligned}
& \frac{\mathrm{d} n_{R}^{*}}{\mathrm{~d} t^{*}}=-(1+\phi) \frac{n_{R}^{*}}{n_{R}^{*}+n_{P}^{*}}+\frac{1}{K_{\mathrm{eq}}} \frac{n_{P}^{*}}{n_{R}^{*}+n_{P}^{*}} \\
& \text { at } t^{*}=0, \quad n_{R}^{*}=1, \\
& \frac{\mathrm{d} n_{P}^{*}}{\mathrm{~d} t^{*}}=\frac{n_{R}^{*}}{n_{R}^{*}+n_{P}^{*}}-\left(\frac{1}{K_{\mathrm{eq}}}+\alpha \phi\right) \frac{n_{P}^{*}}{n_{R}^{*}+n_{P}^{*}}
\end{aligned}
$$

$$
\text { at } t^{*}=0, \quad n_{P}^{*}=0 .
$$


The composition of the permeate stream in the reactor/separator may be calculated through

$\frac{\mathrm{d} \hat{n}_{R}^{*}}{\mathrm{~d} t^{*}}=\phi \frac{n_{R}^{*}}{n_{R}^{*}+n_{P}^{*}}$

at $t^{*}=0, \quad \hat{n}_{R}^{*}=0$,

$\frac{\mathrm{d} \hat{n}_{P}^{*}}{\mathrm{~d} t^{*}}=\alpha \phi \frac{n_{P}^{*}}{n_{R}^{*}+n_{P}^{*}}$

$$
\text { at } t^{*}=0, \quad \hat{n}_{P}^{*}=0 \text {. }
$$

Any of Eqs. (22a), (22b), (23a) and (23b) may be replaced by their algebraic combination, viz

$\frac{\mathrm{d} n_{R}^{*}}{\mathrm{~d} t^{*}}+\frac{\mathrm{d} n_{P}^{*}}{\mathrm{~d} t^{*}}+\frac{\mathrm{d} \hat{n}_{R}^{*}}{\mathrm{~d} t^{*}}+\frac{\mathrm{d} \hat{n}_{P}^{*}}{\mathrm{~d} t^{*}}=0$

$$
\text { at } t^{*}=0, \quad n_{R}^{*}+n_{P}^{*}+\hat{n}_{R}^{*}+\hat{n}_{P}^{*}=1
$$

or, after suitable integration, by

$\hat{n}_{P}^{*}=1-n_{R}^{*}-n_{P}^{*}-\hat{n}_{R}^{*}$.

For consistency with the rationale followed in the case of processes involving sequential reaction and separation, it is considered hereafter that processes involving simultaneous reaction and separation will be allowed to proceed only until the same degree of overall recovery of $\mathrm{P}$ as considered before has been reached, i.e. until

$\hat{n}_{P, i}^{*}=\xi n_{P, r}^{*}=\chi n_{P, R O}^{*}=\chi^{2} n_{P, \text { eq }}^{*}=\chi^{2} K_{\text {eq }} /\left(1+K_{\text {eq }}\right)$,

where subscript $i$ denotes the end of the integrated reaction and separation steps, and where again $\xi$ was considered to be equal to $\chi$. In the case where the molar inventory in the retentate stream vanishes when $t^{*}$ becomes infinite without achievement of the desired degree of recovery of $\mathrm{P}$, then the degree of approach of said situation, $\eta$, will be calculated according to $\eta=\hat{n}_{P, i}^{*} /\left(\chi^{2} n_{P, \text { eq }}^{*}\right)$. In order to facilitate comparison of the performance of the integrated configuration with its sequential counterpart, the variation of $x_{P}^{*}$ and $\hat{x}_{P}^{*}$ with $t^{*}$ until $t_{i}^{*}$ is reached is also depicted in Figs. 3a-c, under the assumptions that $K_{\text {eq }}=1$ and $\chi=\xi=0.95$, and for different combinations of parameters $\phi$ and $\alpha$. Two lines are again plotted, the upper representing the variation of $\hat{x}_{P}^{*}$ in the permeate stream and the lower representing the variation of $x_{P}^{*}$ in the retentate stream.

\section{Thermodynamic evolution}

\subsection{Sequential reaction and separation}

\subsubsection{Reaction step}

The total Gibbs' free energy, $G$, associated with a single-phase binary system (composed of $\mathrm{R}$ and $\mathrm{P}$ only) at any given mole composition and at constant temperature and pressure can be obtained in differential form through the fundamental property relation (Smith and van Ness, 1987), viz.

$$
\begin{aligned}
(\mathrm{d} G= & \left(g_{R}^{\theta}+R T \ln \left\{x_{R}\right\}\right) \mathrm{d} n_{R} \\
& \left.+\left(g_{P}^{\theta}+R T \ln \left\{x_{P}\right\}\right) \mathrm{d} n_{P}\right)_{P, T},
\end{aligned}
$$

where $g_{R}^{\theta}$ and $g_{P}^{\theta}$ denote the molar Gibbs' free energy of pure reactant and product, respectively, at the given absolute temperature $(T)$ and pressure $(P)$, and $R$ denotes the ideal gas constant. Integration of Eq. (26) from the state of pure $R$ to each particular composition of the single stream throughout the reactor leads to

$$
G=n_{R} g_{R}^{\theta}+n_{P} g_{P}^{\theta}+R T\left(n_{R}+n_{P}\right)\left(x_{R} \ln \left\{x_{R}\right\}+x_{P} \ln \left(x_{P}\right\}\right)
$$

which may be rewritten as

$$
\begin{aligned}
G= & n_{0} g_{R}^{\theta}+n_{P} \Delta g_{r}^{\theta} \\
& +R T\left(n_{R}+n_{P}\right)\left(\frac{n_{R}}{n_{R}+n_{P}} \ln \left\{\frac{n_{R}}{n_{R}+n_{P}}\right\}\right. \\
& \left.+\frac{n_{P}}{n_{R}+n_{P}} \ln \left\{\frac{n_{P}}{n_{R}+n_{P}}\right\}\right),
\end{aligned}
$$

where $\Delta g_{r}^{\theta}$ denotes the standard molar Gibbs' free energy of reaction. Recalling the definition of the equilibrium constant at a given temperature, viz. $K_{\text {eq }}=\exp \left\{-\Delta g_{r}^{\theta} / R T\right\}$ (Smith and Van Ness, 1987), recalling the $1: 1$ stoichiometry of the reaction (which implies that $n_{R}+n_{P}=n_{0}$ at all times in the reactor), and using Eq. (5), Eq. (28) finally becomes

$\Delta g^{*}=-n_{P}^{*} \ln \left\{K_{\mathrm{eq}}\right\}+n_{R}^{*} \ln \left\{n_{R}^{*}\right\}+n_{P}^{*} \ln \left\{n_{P}^{*}\right\}$,

where the dimensionless molar Gibbs' free energy of reaction, $\Delta g^{*}$, is defined as

$\Delta g^{*}=\frac{\Delta G}{R T n_{0}}=\frac{G-n_{0} g_{R}^{\theta}}{R T n_{0}}$,

and where variables $n_{R}^{*}$ and $n_{P}^{*}$ are obtained from Eqs. (6) and (7).

Again, it is considered that reaction will be allowed to proceed only until a predefined neighbourhood of equilibrium is reached, i.e. until $n_{P, r}^{*}=\chi n_{P}^{*}$, eq ; the time it will take to achieve such extent is given by Eq. (9).

\subsubsection{Separation step}

In the case of the separation system, a similar integration of Eq. (26) from the state of pure R to each set of particular compositions of the two streams obtained throughout the separator leads to

$$
\begin{aligned}
G= & n_{R} g_{R}^{\theta}+n_{P} g_{P}^{\theta}+R T\left(n_{R}+n_{P}\right)\left(x_{R} \ln \left\{x_{R}\right\}+x_{P} \ln \left\{x_{P}\right\}\right) \\
& +\hat{n}_{R} g_{R}^{\theta}+\hat{n}_{P} g_{P}^{\theta}+R T\left(\hat{n}_{R}+\hat{n}_{P}\right)\left(\hat{x}_{R} \ln \left\{\hat{x}_{R}\right\}\right. \\
& \left.+\hat{x}_{P} \ln \left\{\hat{x}_{P}\right\}\right),
\end{aligned}
$$


where $\hat{n}_{R}$ and $\hat{n}_{P}$ are the numbers of moles of $\mathrm{R}$ and $\mathrm{P}$, respectively, in the permeate stream; Eq. (31) can be rewritten as

$$
\begin{aligned}
G= & \left(n_{R}+n_{P}\right) g_{R}^{\theta}+n_{P} \Delta g_{r}^{\theta} \\
& +R T\left(n_{R}+n_{P}\right)\left(\frac{n_{R}}{n_{R}+n_{P}} \ln \left\{\frac{n_{R}}{n_{R}+n_{P}}\right\}\right. \\
& \left.+\frac{n_{P}}{n_{R}+n_{P}} \ln \left\{\frac{n_{P}}{n_{R}+n_{P}}\right\}\right) \\
& +\left(\hat{n}_{R}+\hat{n}_{P}\right) g_{R}^{\theta}+\hat{n}_{P} \Delta g_{r}^{\theta} \\
& +R T\left(\hat{n}_{R}+\hat{n}_{P}\right)\left(\frac{\hat{n}_{R}}{\hat{n}_{R}+\hat{n}_{P}} \ln \left\{\frac{\hat{n}_{R}}{\hat{n}_{R}+\hat{n}_{P}}\right\}\right. \\
& \left.+\frac{\hat{n}_{P}}{\hat{n}_{R}+\hat{n}_{P}} \ln \left\{\frac{\hat{n}_{P}}{\hat{n}_{R}+\hat{n}_{P}}\right\}\right) .
\end{aligned}
$$

Recalling again the definition of the equilibrium constant and coupling with Eqs. (5) and (9), Eq. (32) finally becomes

$$
\begin{aligned}
\Delta g^{*}= & -\frac{\chi K_{\mathrm{eq}}}{1+K_{\mathrm{eq}}} \ln \left\{K_{\mathrm{eq}}\right\}+n_{R}^{*} \ln \left\{\frac{n_{R}^{*}}{n_{R}^{*}+n_{P}^{*}}\right\} \\
& +n_{P}^{*} \ln \left\{\frac{n_{P}^{*}}{n_{R}^{*}+n_{P}^{*}}\right\}+\hat{n}_{R}^{*} \ln \left\{\frac{\hat{n}_{R}^{*}}{\hat{n}_{R}^{*}+\hat{n}_{P}^{*}}\right\} \\
& +\hat{n}_{P}^{*} \ln \left\{\frac{\hat{n}_{P}^{*}}{\hat{n}_{R}^{*}+\hat{n}_{P}^{*}}\right\},
\end{aligned}
$$

where the variables $n_{P}^{*}, n_{R}^{*}, \hat{n}_{R}^{*}$, and $\hat{n}_{P}^{*}$ are obtained from Eqs. (16b), (18), (19a) and (19b) respectively. Following the same rationale as before, one may assume that separation will be allowed to proceed only until a predefined neighbourhood of total recovery of $\mathrm{P}$ is reached, i.e. $\hat{n}_{P, s}^{*}=\xi n_{P, r}^{*}=\chi n_{P, r}^{*}$; the time required for such operation is given by Eq. (20).

For the reactor/separator sequential configuration, the variation of the dimensionless Gibbs' molar free energy of reaction, $\Delta g^{*}$, is represented in Figs. $4 \mathrm{a}-\mathrm{c}$ as a function of the dimensionless time, $t^{*}$, under the assumptions that $K_{\text {eq }}=1$ and that reaction proceeds until $95 \%$ of the equilibrium conversion is attained, i.e. $\chi=0.95$. For values of $t^{*}$ above $t_{r}^{*}$, the variation of $\Delta g^{*}$ with $t^{*}$ is represented in the same plots, again under the assumption that $\xi=\chi=0.95$, for different combinations of parameters $\phi$ and $\alpha$.

\subsection{Simultaneous reaction and separation}

In the case of the system that integrates reaction and separation, integration of Eq. (26) from the state of pure $\mathrm{R}$ to each set of particular compositions of the two streams obtained throughout the reactor/separator leads to

$$
\begin{aligned}
G= & n_{R} g_{R}^{\theta}+n_{P} g_{P}^{\theta}+R T\left(n_{R}+n_{P}\right)\left(x_{R} \ln \left\{x_{R}\right\}+x_{P} \ln \left\{x_{P}\right\}\right) \\
& +\hat{n}_{R} g_{R}^{\theta}+\hat{n}_{P} g_{P}^{\theta}+R T\left(\hat{n}_{R}+\hat{n}_{P}\right)\left(\hat{x}_{R} \ln \left\{\hat{x}_{R}\right\}\right. \\
& \left.+\hat{x}_{P} \ln \left\{\hat{x}_{P}\right\}\right),
\end{aligned}
$$

which may be rewritten as

$$
\begin{aligned}
G= & \left(n_{R}+n_{P}\right) g_{R}^{\theta}+n_{P} \Delta g_{r}^{\theta} \\
& +R T\left(n_{R}+n_{P}\right)\left(\frac{n_{R}}{n_{R}+n_{P}} \ln \left\{\frac{n_{R}}{n_{R}+n_{P}}\right\}\right. \\
& \left.+\frac{n_{P}}{n_{R}+n_{P}} \ln \left\{\frac{n_{P}}{n_{R}+n_{P}}\right\}\right) \\
& +\left(\hat{n}_{R}+\hat{n}_{P}\right) g_{R}^{\theta}+\hat{n}_{P} \Delta g_{r}^{\theta} \\
& +R T\left(\hat{n}_{R}+\hat{n}_{P}\right)\left(\frac{\hat{n}_{R}}{\hat{n}_{R}+\hat{n}_{P}} \ln \left\{\frac{\hat{n}_{R}}{\hat{n}_{R}+\hat{n}_{P}}\right\}\right. \\
& \left.+\frac{\hat{n}_{P}}{\hat{n}_{R}+\hat{n}_{P}} \ln \left\{\frac{\hat{n}_{P}}{\hat{n}_{R}+\hat{n}_{P}}\right\}\right) .
\end{aligned}
$$

Recalling again the definition of the equilibrium constant and coupling with Eq. (5), Eq. (35) finally becomes

$$
\begin{aligned}
\Delta g^{*}= & -\left(n_{P}^{*}+\hat{n}_{P}^{*}\right) \ln \left\{K_{\mathrm{eq}}\right\}+n_{R}^{*} \ln \left\{\frac{n_{R}^{*}}{n_{R}^{*}+n_{P}^{*}}\right\} \\
& +n_{P}^{*} \ln \left\{\frac{n_{P}^{*}}{n_{R}^{*}+n_{P}^{*}}\right\}+\hat{n}_{R}^{*} \ln \left\{\frac{\hat{n}_{R}^{*}}{\hat{n}_{R}^{*}+\hat{n}_{P}^{*}}\right\} \\
& +\hat{n}_{P}^{*} \ln \left\{\frac{\hat{n}_{P}}{\hat{n}_{R}+\hat{n}_{P}^{*}}\right\},
\end{aligned}
$$

where the variables $n_{R}^{*}, n_{P}, \hat{n}_{R}^{*}$ and $\hat{n}_{P}^{*}$ are obtained from Eqs. (22a), (22b), (23a) and (23b) respectively. Again, processes involving simultaneous reaction and separation will be allowed to proceed only until the same degree of overall recovery of $\mathrm{P}$ as considered before has been reached, i.e. $\hat{n}_{P, i}^{*}=\xi \quad n_{P, r}^{*}=\chi n_{P, r}^{*}=\chi^{2} n_{P \text {, eq }}^{*}=\chi^{2} K_{\text {eq }} /$ $\left(1+K_{\text {eq }}\right)$.

As done above, in order to facilitate comparison of the performance of the integrated configuration with its sequential counterpart, the variation of $\Delta g^{*}$ with $t^{*}$ until $t_{i}^{*}$ is reached is also depicted in Figs. $4 \mathrm{a}-\mathrm{c}$, under the assumptions that $K_{\text {eq }}=1$ and that $\chi=\xi=0.95$, for different combinations of parameters $\phi$ and $\alpha$.

\section{Discussion}

The theoretical development presented above has attempted to, in an analytical way, compare the performance of a sequential process configuration (where reaction is followed by separation), with that of an integrated process configuration, (where reaction and separation 

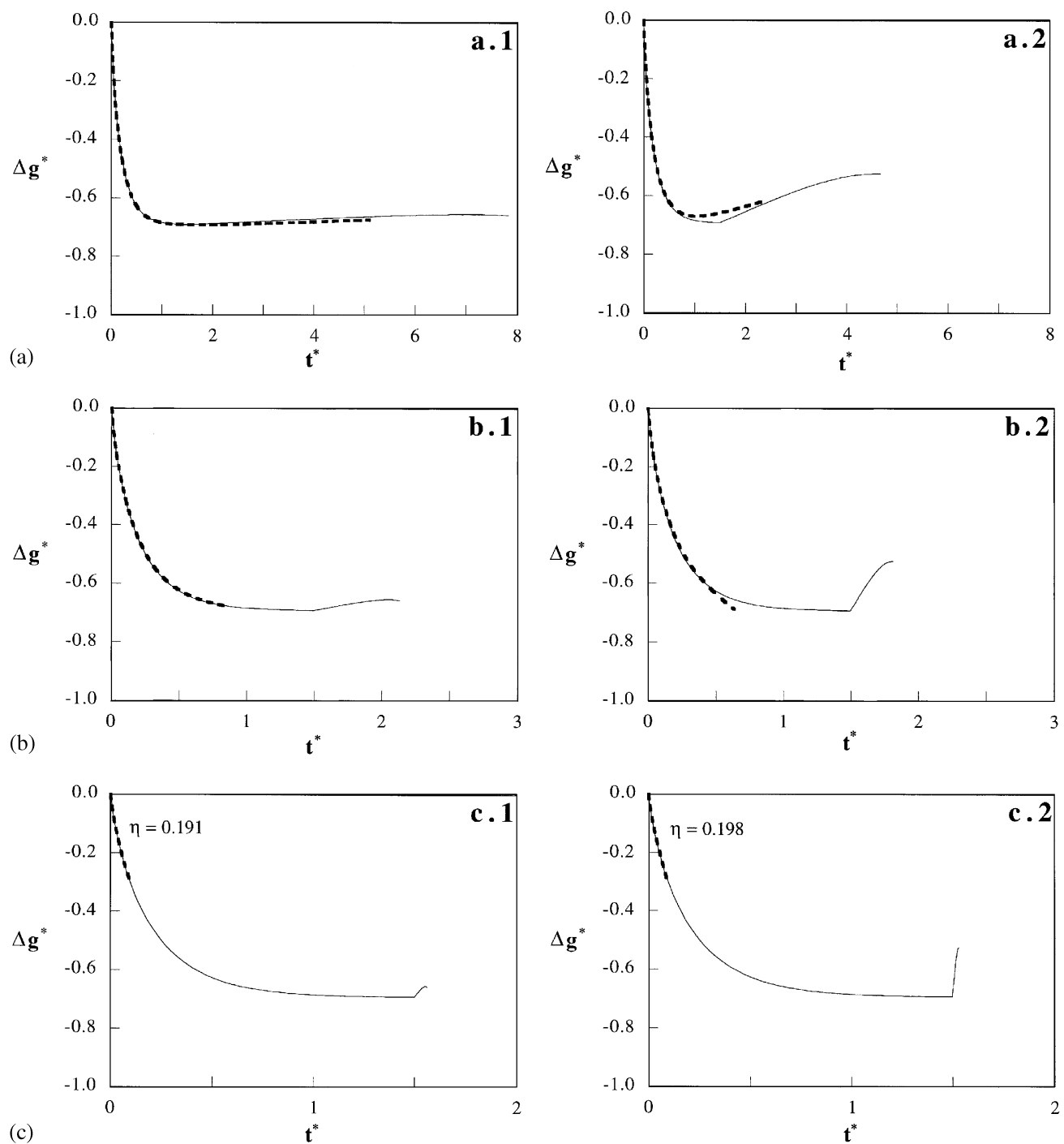

Fig. 4. Thermokinetic profiles at the outlets of the reactor and separator in the case of sequential reaction and separation ( -$)$, or at the outlet of the integrated unit in the case of simultaneous reaction and separation (-- ), expressed as dimensionless molar Gibbs' free energy of reaction, $\Delta g^{*}$, vs. the dimensionless time, $t^{*}$, for $K_{\text {eq }}=1$ and (a) $\phi=0.1$, (b) $\phi=1$ and (c) $\phi=10$, and for (1) $\alpha=2$ and (2) $\alpha=5$.

occur simultaneously), both in kinetic and in thermodynamic terms. In order to do so, a rather simple model system had to be postulated, which consists of a reversible reaction, following a $1: 1$ stoichiometry, that evolves from pure reactant to a thermodynamically ideal reactant/product homogeneous mixture. For the sake of simplicity, such reaction was assumed to follow first-order kinetics, which eventually implies that conclusions obtained from this study may not be applicable in a straightforward fashion to other situations where nonlinear kinetics prevails. Although it might be argued that, for linear systems, the principles of superposition often dictate that changes in configuration lead to identical performance, as is the notable case of a cascade of a plug flow reactor and a well mixed reactor (which performs identically irrespective of the relative position of the units), that does not necessarily extrapolate to a cascade of a plug flow reactor and a separator; in fact, the total number of moles is conserved in the reactor between inlet and outlet streams, but the same does not apply in the separator between inlet and one of the outlet (i.e. permeate or retentate) streams, so an intrinsic non-linearity is already present; that might possibly make the performance of the overall system less dependent on whether linear or non-linear reaction kinetics are considered.

Parameters claimed to play a role throughout this study comprise:

(i) two reactional parameters, viz. the equilibrium constant, $K_{\text {eq }}$ (which describes thermodynamic inhibition); and the degree of completion of production of 
$\mathrm{P}, \chi$ (which measures approach to equilibrium conditions); and

(ii) three separational parameters, viz. the ratio of the time scale associated with mass transfer of $\mathrm{R}$ (i.e. $v n_{0} / k_{R}$ ) to the time scale associated with mass transfer of $\mathrm{P}$ (i.e. $v n_{0} / k_{P}$ ), $\alpha$ (which compares the rates of removal of product and reactant in the separator); the ratio of the time scale associated with chemical reaction (i.e. $v n_{0} / k_{1}$ ) to the time scale associated with mass transfer of $\mathrm{R}$ (i.e. $v n_{0} / k_{R}$ ), $\phi$ (which compares the rates of disappearance of $\mathrm{R}$ by chemical reaction and by physical interfacial transport); and the degree of completion of recovery of product, $\xi$ (which compares the fraction of product recovered in the permeate stream with the amount of product that enters the separator). In this way, the operating regime can be considered as kinetically controlled (for large $\phi$ ) or diffusionally controlled (for small $\phi$ and large $\alpha$ : control of R; for small $\phi$ and small $\alpha$ : control of P).

\subsection{Kinetic evolution}

Results concerning the kinetic evolution in both configurations studied (sequential and integrated) are represented in Figs. $3 \mathrm{a}-\mathrm{c}$ as the variation of the molar fraction of $\mathrm{P}$ (in both retentate, $x_{P}^{*}$, and permeate, $\hat{x}_{P}^{*}$, streams) as a function of time $\left(t^{*}\right)$. In the following analysis both the fractional amount of $\mathrm{P}$ and time are important; the first because production of a desired product is the objective of any reactional process, and the second because time plays the role of a currency in the kinetic domain, i.e. time is the price to be paid if one wants to bring about actual phenomena that proceed at finite rates. Variable $t^{*}$ may be viewed as the processing time normalized by the time scale associated with chemical reaction in the forward direction (i.e. $v n_{0} / k_{1}$ ).

For all plots, $K_{\text {eq }}$ was assumed to be equal to unity (note that other situations, not shown, were also analysed), $\phi$ was assumed to take values of 0.1 (Fig. 3a), 1 (Fig. 3b) and 10 (Fig. 3c) (thus spanning the whole range between diffusional and kinetic control), and $\alpha$ was assumed to take values of 2 (Figs. 3a.1, b.1 and c.1) and 5 (Figs. 3a.2, b.2 and c.2). In these plots the variation of the molar fractional amount of $P$ is represented by thin lines in the case of the sequential configuration and by thick lines in the case of the integrated one. As can be seen from inspection of the plots, in the case of the sequential configuration, one single line exists until $t_{r}^{*}$ (which is only a function of $\chi$ and $K_{\text {eq }}$ ) is reached (representing the variation of the molar fraction of $\mathrm{P}$ produced inside the reactor) and two lines for values of $t^{*}$ higher than $t_{r}^{*}$ : the upper $(----)$ represents the dimensionless molar fraction of $\mathrm{P}$ in the permeate stream, $\hat{x}_{P}^{*}$ (which is richer in $\mathrm{P}$ ) and the lower (- $\longrightarrow$ ) represents the dimensionless molar fraction of $\mathrm{P}$ in the retentate stream, $x_{P}^{*}$ (which is poorer in $\mathrm{P}$ ). In the case of the integrated configuration, where separation takes place simultaneously with reaction, two (thick) lines exist right from the beginning, and again the upper (-----) represents the dimensionless molar fraction of $\mathrm{P}$ in the permeate stream, $\hat{x}_{P}^{*}$ (which is richer in $\mathrm{P}$ ) and the lower (-) represents the dimensionless molar fraction of $\mathrm{P}$ in the retentate stream, $x_{P}^{*}$ (which is poorer in $\mathrm{P}$ ).

Although, for the sake of bookkeeping, plots are only presented for $K_{\mathrm{eq}}=1$, it was observed that, for $\chi=0.95$, $t_{r}^{*}$ and $x_{P, r}^{*}$ increase as $K_{\text {eq }}$ increases, hence implying, as expected, that longer times are required to reach the corresponding higher equilibrium conversions. It is possible to observe that, in all situations, the molar fraction of $\mathrm{P}$ in the retentate stream $\left(x_{P}^{*}\right)$ goes through a maximum at $t_{r}^{*}$ and decreases thereafter, whereas the molar fraction of $\mathrm{P}$ in the permeate stream $\left(\hat{x}_{P}^{*}\right)$ displays a discontinuity towards a higher concentration (due to the postulated preferential removal of P, i.e. $\alpha>1$ ) followed by a decrease thereafter; this means that the local maxima of both $x_{P}^{*}$ and $\hat{x}_{P}^{*}$ coincide with the composition at the very outlet of the reactor. The molar fractions in both these streams are now not only a function of $\chi$ and $K_{\text {eq }}$, but also a function of $\phi$ and $\alpha$. The definition of all such parameters and variables was, as already mentioned, done so as to enhance their physicochemical meaning.

Inspection of the plots depicted in Fig. 3 also indicates that, when reaction and separation occur simultaneously, the variation of the molar fraction of $\mathrm{P}$ is again represented by two lines, one corresponding to the permeate and another corresponding to the retentate streams of the integrated unit; however, unlike the previous situation, they now both start at time zero because separation and reaction are indissociable and, as expected, $\hat{x}_{P}^{*}$ is always higher than $x_{P}^{*}$. Both molar fractions are dependent on parameters $\chi, \phi, K_{\text {eq }}$ and $\alpha$, and, in general, $x_{P}^{*}$ undergoes a fast increase at short times and then tends to an asymptotic plateau. It should be noted that, in the classical unit operation approach, the reactant disappears from the reactor only by chemical reaction; when the integrated system is considered, reaction and separation occur at the same time, so reactant disappears from the system not only by chemical reaction but also by withdrawal to the permeate stream, thus making it unavailable to further react. Therefore, the results obtained for the integrated system may be worse than those obtained for the unit operation approach, as emphasized by the values of $\eta$ below unity in Figs. 3c.1 and c.2 (i.e. in those plots corresponding to high values of $\phi$ ). Similar trends were also observed for other values of $K_{\text {eq }}$ tested. Therefore, integration of reaction and separation should not be sought if the mass transfer coefficient for the reactant is much higher than that for the product (say $\phi>5$ ).

As a general conclusion, it can be stated that, from a kinetic point of view, simultaneous reaction and separation should give rise to better results than sequential reaction and separation because the overall mole 
inventory in the former case decreases continuously at the expense of preferential removal of product $\mathrm{P}$, i.e. integration promotes increase in $C_{R}$ and decrease in $C_{P}$ when compared with plain reaction for the same reaction extent; so, the rate of the forward reaction is favoured and the rate of the reverse reaction is unfavoured. Furthermore, since continuous removal of product $\mathrm{P}$ does not allow chemical equilibrium to be ever reached, the rate of reaction in the reverse direction is further decreased. However, this is not always observed in the plots generated. (Comparison of the relative performance of the two approaches is based on the curves which represent the compositions of the two outlet streams of the separator in the unit operation configuration with the curves which represent the compositions of the two outlet streams of the reactor/separator in the integrated approach, both at any given time $t^{*}$.) In all situations, the time required by the integrated system to achieve the preset degree of conversion and separation is always much lower than the one required to achieve the same conversion and separation in the classical system; there are cases where $t_{i}^{*}$ is even lower than $t_{r}^{*}$. The time frame for the sequential reaction and separation is shortened when parameter $\phi$ increases (which is a consequence of faster overall mass transfer) and parameter $\alpha$ increases (which is a consequence of faster transport of $P$ relative to $R$ ), both situations where recovery of $P$ is kinetically controlled. For low values of $K_{\text {eq }}$ and $\phi$, the final molar fraction of product in the retentate stream is always higher in the case of the integrated system than in the sequential one; such trend is reversed for high values of $K_{\mathrm{eq}}$ and $\phi$. In terms of the composition of the permeate stream (i.e. the stream with commercial value), the final molar fraction of product is always higher in the integrated configuration than in the sequential counterpart, except when $\phi$ is high (as seen before). The effect of $\phi$ is stronger for higher values of $K_{\text {eq }}$, irrespective of the actual value of $\alpha$; however, for the same values of $K_{\text {eq }}$ and $\phi$, a higher $\alpha$ leads to wider differences between intermediate and final compositions of $\mathrm{P}$ in both streams, for both systems. When the remaining parameters are held constant, higher values of $K_{\text {eq }}$ lead, as expected, to higher intermediate and final molar fractions of $\mathrm{P}$ in both streams and for both configurations under scrutiny. With respect to $\phi$, it is observed that when the remaining parameters are held constant, the molar fractions of $\mathrm{P}$ in the classical system are not much affected; on the contrary, the molar fractions in the integrated system are lower for higher values of $\phi$.

\subsection{Thermodynamic evolution}

Results concerning the thermodynamic evolution in both configurations studied (sequential and integrated) are represented in Figs. $4 \mathrm{a}-\mathrm{c}$ as the variation of the dimensionless molar Gibbs' free energy $\left(\Delta g^{*}\right)$ as a func- tion of the dimensionless time $\left(t^{*}\right)$. In the sequential configuration the behaviour of the system is represented by a single line (-), both before and after $t_{r}^{*}$. Such realization arises from the fact that $\Delta g^{*}$ encompasses the compositions of both outlet streams of the separation unit. Prior to $t_{r}^{*}, \Delta g^{*}$ is only a function of $\chi$ and $K_{\text {eq }}$, and for all plots there is always a minimum for $\Delta g^{*}$ corresponding to the time at which the reaction stopped (i.e. at the preset $95 \%$ conversion), irrespective of the actual values of parameters $\alpha$ and $\phi$. For the case of the integrated approach, the thermodynamic behaviour (also represented in Figs. $4 \mathrm{a}-\mathrm{c}$ ) is again represented by one single line (-..-), which depends on the values of $\chi, \phi$, $K_{\text {eq }}$ and $\alpha$. The general trend is a high decrease of $\Delta g^{*}$ at initial times which is followed afterwards by either a clear increase or a gradual tendency to steady values, depending on the actual values chosen for parameters $\phi, K_{\text {eq }}$ and $\alpha$. In either case, the initial evolution (i.e. at low processing times) of $\Delta g^{*}$ for the integrated configuration is virtually coincident with that for the classical one.

One can also observe that the trend followed by both systems is similar for low processing times and consists on a very sharp decrease in $\Delta g^{*}$ (which always assumes negative values, meaning, as expected, that reaction is a spontaneous process) until a minimum of $\Delta g^{*}$ is reached (which corresponds to a preset vicinity of equilibrium). For the integrated unit, such minimum may not ever be reached when high values of $\phi$ are considered; when it does (e.g. at $\phi=0.1$ ), it occurs at the same time (for low $\alpha$ ) or before the time (for high $\alpha$ ) obtained using the classical configuration. For the unit operation approach, as separation begins there is consistently an increase in $\Delta g^{*}$ until eventually a local maximum is reached; from then on, the curve tends again to the aforementioned minimum value of $\Delta g^{*}$ after a finite time, that can be calculated from Eq. (18) after setting $n_{R}^{*}=0$, i.e.

$t^{*}=\frac{K_{\mathrm{eq}}}{\phi\left(1+K_{\mathrm{eq}}\right)}\left(\frac{\chi}{\alpha}+\frac{1}{K_{\mathrm{eq}}}+1-\chi-\phi \ln \{1-\chi\}\right)$.

This behaviour is expected because part of the Gibbs' free energy input to the system undergoing separation returns again to the surroundings because the P-rich stream thus generated is mixed with the P-poor stream that was already separated; as recovery of $\mathrm{P}$ approaches completion, the retentate will disappear whereas the permeate will have the same composition as if there had been no separation at all, which, in terms of Gibbs' free energy, is equivalent to the aforementioned minimum associated with chemical equilibrium. In the situation where reaction and separation occur sequentially, this observation does not hold because the state of quasiequilibrium that would correspond to startup of the separation process in the unit operation approach will never be recovered since the overall system has been already forced past equilibrium. 
Although not shown, $K_{\text {eq }}$ does not seem to have a clear influence on the results when the remaining parameters are held constant; however, higher values of $\phi$ apparently lead to higher values of $\Delta g^{*}$. Eq. (33) may be rewritten as

$$
\begin{aligned}
\Delta g^{* *}= & -\frac{\chi K_{\mathrm{eq}}}{1+K_{\mathrm{eq}}} \ln \left\{K_{\mathrm{eq}}\right\}+\ln \left\{1+K_{\mathrm{eq}}\right\} \\
& +n_{R}^{*} \ln \left\{n_{R}^{*}\right\}+n_{P}^{*} \ln \left\{n_{P}^{*}\right\}+\hat{n}_{R}^{*} \ln \left\{\hat{n}_{R}^{*}\right\} \\
& +\hat{n}_{P}^{*} \ln \left\{\hat{n}_{P}^{*}\right\} \\
& -\left(n_{R}^{*}+n_{P}^{*}\right) \ln \left\{n_{R}^{*}+n_{P}^{*}\right\}-\left(\hat{n}_{R}^{*}+\hat{n}_{P}^{*}\right) \\
& \times \ln \left\{\hat{n}_{R}^{*}+\hat{n}_{P}^{*}\right\},
\end{aligned}
$$

where $\Delta g^{* *}$ is defined as

$\Delta g^{* *}=\Delta g^{*}-\Delta g_{\mathrm{eq}}^{*}$

and where advantage was taken from Eqs. (8) and (29). Inspection of these two equations indicates that the contribution arising from the separation step towards the overall Gibbs' free energy can be decomposed into a set of negative contributions (viz. $n_{R}^{*} \ln \left\{n_{R}^{*}\right\}, n_{P}^{*} \ln \left\{n_{P}^{*}\right\}$, $\hat{n}_{R}^{*} \ln \left\{\hat{n}_{R}^{*}\right\}$ and $\left.\hat{n}_{P}^{*} \ln \left\{\hat{n}_{P}^{*}\right\}\right)$, and a set of positive contributions (viz. $-\left(n_{R}^{*}+n_{P}^{*}\right) \ln \left\{n_{R}^{*}+n_{P}^{*}\right\}$ and $-\left(\hat{n}_{R}^{*}+\hat{n}_{P}^{*}\right)$ $\left.\ln \left\{\hat{n}_{R}^{*}+\hat{n}_{P}^{*}\right\}\right)$. This means that the composition effects are favourable (in terms of spontaneity of the overall process) whereas the volume-reduction effects are unfavourable (a consequence of the fact that overall volume reductions due to preferential migration of components are not spontaneous).

Although the analysis developed implicitly assumed reversible processes, it should be noted that the total variation of Gibbs' free energy that occurs during separation is equal to the actual work supplied to the system only in such case; for real (and hence irreversible) processes, the total Gibbs' free energy of separation will be below the separation work, and this fact should be taken into account in extrapolations of these conclusions to situations of practical interest.

\section{Acknowledgements}

Partial funding for this research work was provided through grant PRAXIS/2/2.1/BIO/34/94 (Portugal, program PRAXIS XXI, Project Process Integration: Extractive Biocatalysis). Funding for author Ana L. Paiva was provided through $\mathrm{Ph} . \mathrm{D}$. fellowships BD/2081/92-IF and BD $/ 5568 / 95$ (Portugal, programs CIENCIA and PRAXIS XXI). Funding for author Diman van Rossum was provided through a postdoctoral fellowship ERBCHBGCT940591 EU, program Human Capital and Mobility, project BIOCAD-BIOCAtalytic Distillation).

\section{Notation}

$C_{P} \quad$ molar concentration of $\mathrm{P}, \mathrm{mol} \mathrm{m}^{-3}$

$C_{P \text {, eq }} \quad$ value of $C_{P}$ at chemical equilibrium, $\mathrm{mol} \mathrm{m}^{-3}$

$C_{R}$

$C_{R, \text { eq }}$

$g_{P}^{\theta}$

$g_{R}^{\theta}$

G

$k_{1}$

$k_{-1}$

$k_{P}$

$k_{R}$

$K_{\text {eq }}$

$n_{0}$

$n_{P}$

$n_{P}^{*}$

$n_{P, \text { eq }}^{*}$

$\hat{n}_{P}$

$\hat{n}_{P}^{*}$

$n_{P, i}$

$n_{P, i}^{*}$

$\hat{n}_{P, i}$

$\hat{n}_{P, i}^{*}$

$n_{P, r}$

$n_{P, r}^{*}$

$n_{P, s}^{*}$

$\hat{n}_{P, s}^{*}$

$n_{R}$

$n_{R}^{*}$

$\hat{n}_{R}$

$\hat{n}_{R}^{*}$

$n_{R \text {, eq }}^{*}$

$n_{R, i}$

$n_{R, i}^{*}$

$\hat{n}_{R, i}$

$\hat{n}_{R, i}^{*}$

$n_{R, r}^{*}$

$n_{R, s}^{*}$

molar concentration of $\mathrm{R}, \mathrm{mol} \mathrm{m}^{-3}$

value of $C_{R}$ at chemical equilibrium, $\mathrm{mol} \mathrm{m}^{-3}$ molar Gibbs' free energy of $\mathrm{P}, \mathrm{J} \mathrm{mol}^{-1}$ molar Gibbs' free energy of $\mathrm{R}, \mathrm{J} \mathrm{mol}^{-1}$ overall Gibbs' free energy, $\mathrm{J}$

first-order kinetic constant describing rate of forward reaction, $\mathrm{m}^{3} \mathrm{~s}^{-1}$

first-order kinetic constant describing rate of reverse reaction, $\mathrm{m}^{3} \mathrm{~s}^{-1}$

first-order kinetic constant describing rate of mass transfer of $\mathrm{P}, \mathrm{m}^{3} \mathrm{~s}^{-1}$

first-order kinetic constant describing rate of mass transfer of $\mathrm{R}, \mathrm{m}^{3} \mathrm{~s}^{-1}$

equilibrium constant, dimensionless

initial number of moles, mol

number of moles of $\mathrm{P}$, mol

normalized counterpart of $n_{P}$, dimensionless

value of $n_{P}^{*}$ at chemical equilibrium, dimensionless

number of moles of $\mathrm{P}$ in permeate stream, mol normalized counterpart of $\hat{n}_{P}$, dimensionless number of moles of $\mathrm{P}$ in retentate stream at the outlet of the integrated unit, mol

normalized counterpart of $n_{P, i}$, dimensionless number of moles of $\mathrm{P}$ in permeate stream at the outlet of the integrated unit, dimensionless

normalized counterpart of $\hat{n}_{P, i}$, dimensionless number of moles of $\mathrm{P}$ at the outlet of the reaction unit, mol

normalized counterpart of $n_{P, r}$, dimensionless value of $n_{P}^{*}$ at the outlet of the separation unit, dimensionless

value of $\hat{n}_{P}^{*}$ at the outlet of the separation unit, dimensionless

number of moles of $\mathrm{R}$, mol

normalized counterpart of $n_{R}$, dimensionless number of moles of $\mathrm{R}$ in permeate stream, mol

normalized counterpart of $\hat{n}_{R}$, dimensionless value of $n_{R}^{*}$ at chemical equilibrium, dimensionless

number of moles of $\mathrm{R}$ in retentate stream at the outlet of the integrated unit, mol

normalized counterpart of $n_{R, i}$, dimensionless number of moles of $\mathrm{R}$ in permeate stream at the outlet of the integrated unit, mol normalized counterpart of $\hat{n}_{R, i}$, dimensionless value of $n_{R}^{*}$ at the outlet of the reaction unit, dimensionless

value of $n_{R}^{*}$ at the outlet of the separation unit, dimensionless 
pressure, $\mathrm{Pa}$

product, dimensionless

ideal gas constant, $\mathrm{J} \mathrm{mol}^{-1} \mathrm{~K}^{-1}$

reactant, dimensionless

time elapsed after startup of batch reactor or separator or reactor/separator (or space time of plug-flow reactor or separator or reactor/separator), s

dimensionless counterpart of $t$, dimensionless value of $t$ at the outlet of the integrated unit, $\mathrm{s}$ dimensionless counterpart of $t_{i}$, dimensionless value of $t^{*}$ at the outlet of the reaction unit, dimensionless

value of $t$ at the outlet of the separation unit, s dimensionless counterpart of $t_{s}$, dimensionless

absolute temperature, $\mathrm{K}$

molar volume of either $\mathrm{R}$ or $\mathrm{P}, \mathrm{m}^{3} \mathrm{~mol}^{-1}$

total volume of reaction system, $\mathrm{m}^{3}$

molar fraction of $\mathrm{P}$, dimensionless

molar fraction of $\mathrm{R}$, dimensionless

\section{Greek letters}

ratio of time scales for mass transfer of $\mathrm{R}$ and $\mathrm{P}$, dimensionless

$\Delta g^{*} \quad$ dimensionless variation in Gibbs' molar free energy, dimensionless

$\Delta g^{* *} \quad$ alternative dimensionless variation in Gibbs' molar free energy, dimensionless

$\Delta g_{\mathrm{eq}}^{*} \quad$ value of $\Delta g^{*}$ at chemical equilibrium, dimensionless

$\Delta g_{r}^{\theta} \quad$ standard molar Gibbs' free energy of reaction, $\mathrm{J} \mathrm{mol}^{-1}$

$\Delta G \quad$ variation of overall Gibbs' free energy, $\mathrm{J}$

$\eta \quad$ degree of approach of desired recovery of $\mathrm{P}$, dimensionless $\xi \quad$ degree of completion of recovery of $\mathrm{P}$, dimensionless

$\phi \quad$ ratio of time scales for chemical reaction and mass transfer of $\mathrm{R}$, dimensionless

$\chi$ degree of completion of production of $\mathrm{P}$, dimensionless

\section{References}

Laane, C., Tramper J., \& Lilly, M.D. (Eds.). (1987). Biocatalysis in organic media. Amsterdam, Netherlands: Elsevier.

Paiva, A.L., \& Malcata, F.X. (1994). Process integration involving lipase-catalyzed ester synthesis reactions. Biotechnology Techniques, $8,629-634$.

Paiva, A.L., \& Malcata, F.X. (1996). Integration of reaction and separation with lipases: Biocatalytic distillation. In F.X. Malcata (Ed.), Engineering of/with lipases (pp. 597-611). Dordrecht: Kluwer Academic Publishers.

Paiva, A.L., \& Malcata, F.X. (1997a). Integration of reaction and separation with lipases: An overview. Journal of Molecular Catalysis B: Enzymatic, 3, 99-109.

Paiva, A.L., \& Malcata, F.X. (1997b). Does thermodynamics improve processing when chemical reaction is integrated with physical separation in binary ideal mixtures? Chemical Engineering Science, 52 , 449-452.

Paiva, A.L., \& Malcata, F.X. (1997c). Reversible reaction and diffusion within a porous catalyst slab. Chemical Engineering Science, 52, 4429-4432.

Paiva, A.L., \& Malcata, F.X. (1999). Comparison of the performance of integrated and sequential reaction and separation units in terms of recovery of a desired product. Chemical Engineering Science (submitted).

Paiva, A.L., van Rossum, D., \& Malcata, F.X. (1998). Cascading reactor-separator sets reduces total time for low yield Michaelis Menten reactions: Model predictions. Biocatalysis and Biotransformation, 16, 205-224.

Tramper, J., Vermue, M.H., Beeftink, H.H., \& von Stockar, U. (Eds.). (1992). Biocatalysis in non-conventional media. Amsterdam, Netherlands: Elsevier.

Smith, J.M., \& van Ness, H.C. (Eds.). (1987). Introduction to chemical engineering thermodynamics. New York, USA: McGraw-Hill. 